\section{MAY 2009}

The 58th International Congress of the European Society for Cardiovascular Surgery

Congress: May 1-4, 2009, Warsaw, Poland. For more information, contact: ESCVS General Secretariat, Prof. Claudio Muneretto, ESCVS Secretary General, UDA Cardiochirurgia, Spedali Civili P.le Spedali Civili 1, 25123 Brescia, Italy; (telephone: +39030 3996401; fax: +39 030 3996096; E-mail: escvs. secretariat@gmail.com; Website: http:// www.escvs.org/).

\section{The 8th International}

Symposium on Endovascular

Therapeutics

Symposium: May 7-9, 2009, Hotel Hilton, Barcelona, Spain. Abstract submission deadline: February 28, 2009. For more information, contact: Atlanta Unicongress Calvet 55 (Telephone: +34- 902- 109- 853; Fax: +34902- 109- 855; E-mail: site2009@ unicongress.org).

\section{Eighth International Symposium} on Redo Cardiac Surgery in Adults

Symposium: May 8-9, 2009, in Boston, Massachusetts. For more information, contact: University of Pittsburgh Medical Center, Division of Cardiac Surgery The Heart, Lung, and Esophageal Surgery Institute, Shadyside Medical Building, Suite 7065200 Centre Avenue Pittsburgh, PA 15232 (Telephone: 1- 412- 623-3140; Fax: 1- 412623-2822; E-mail: slomkasm@upmc. edu).

\footnotetext{
Announcements of major meetings and other significant activities must be received at least 8 weeks before the desired month of publication. Information will be limited to title of meeting, date, place, and an address to obtain further information. Send announcements to Ryan Walther, Managing Editor, The Journal of Thoracic and Cardiovascular Surgery, 900 Cummings Center, Suite 221-U, Beverly, MA 01915 (E-mail: rwalther@prri.com).
}

The 55th Annual Conference of the Indian Association of Cardiovascular \& Thoracic Surgeons (CT CON 2009)

Conference: May 14-17, 2009, Srinagar, Kashmir, India. For more information, contact: Conference Secretariat, Prof. AG Ahangar, Head of Department of Cardiovascular \& Thoracic Surgery. Sher-I-Kashmir Institute of Medical Sciences, Soura, Srinagar, India; Tel: +91- 194- 2400348; E-mail: iacts2009@yahoo.com).

\section{European School for Cardio-}

Thoracic Surgery Cardiac Course Level B

Meeting: May 18-23, 2009, Bergamo, Italy. For more information, contact: EACTS Executive Secretariat, 3 Park St., Windsor, Berkshire S14 1LU, UK (Telephone: +44-1753832-166; Fax: +44-1753-620407; E-mail: info@eacts.co.uk).

\section{Postgraduate Graduate Course in General Thoracic Surgery}

Course: May 21-22, 2009, Boston, Massachusetts. For more information: Call: (617) 384-8600, 10:00 am-4:00 pm Eastern Time, Monday-Friday; Fax: (617)384-8686; E-mail:hms-cme@ hms.harvard.edu.

\section{The XIV Congress of the Catalan}

Society for Cardiac Surgery

Congress: May 27-30, 2009, Hotel Avenida Palace, Barcelona Spain. Abstract submission deadline: March 15, 2009. For more information, contact: Nuria Bosch - Oldisseny S.L., Comte (Telephone: +34- 934- 510- 792; Fax: +34- 934- 511- 736; E-mail: nbosch@ oldisseny.com).

\section{5th International Conference on Pediatric Mechanical Circulatory Support Systems \& Pediatric Cardiopulmonary Perfusion \\ Conference: May 27-30, 2009, Dallas, Texas. For more information: pedsabstracts@hmc.psu.edu.Website: http://www.hmc.psu.edu/childrens/ pedscpb/}

ASAIO - IFAO Annual Conference in conjunction with the 5th Annual Pediatric Mechanical Circulatory Support Systems \& Pediatric

Cardiopulmonary Perfusion

Conference: May 28-30, 2009, Hilton Anatole Hotel, Dallas, Texas. For more information, contact: ASAIO Inc., 980 North Federal Highway, Suite 212, Boca Raton, Florida 33432-2711 (Telephone: 1- 561- 3918589; Fax: 1 -561- 368-9153; E-mail: info@asaio.com).

\section{3rd Ukrainian-Polish Congress} of Cardiovascular Surgery

Meeting: May 28-29, 2009, Kyiv, Ukraine. For more information, contact: M.M. Amosov National Institute of Cardiovascular Surgery of the Academy of Medical Sciences of Ukraine, 6 M.Amosov Street, MSP 03680 (Telephone: +38 (0)44- 27543-22; Fax: +38 (0)44 -275-43-22; E-mail: info@amosovinstitute.org.ua).

17th European Conference on General Thoracic Surgery

Conference: May 31 - June 3, 2009, Krakow, Poland. For more information, contact: Mondial Congress and Events, Mondial GrmbH \& Co., Operngasse 20b, 10-40 Vienna, Austria; (telephone: +43 1588040 ; fax +431 588040 185; E-mail: ests2009@ mondial-congress.com; Website: http:// www.estsmeetings.org/2009/).

\section{JUNE 2009}

Fifth Biennial Meeting of The Society for Heart Valve Disease Meeting: June 2009, Ritz Carlton Hotel, Berlin Germany. For information, contact: SHVD Executive Secretariat, Heart Science Centre, Harefield Hospital, Harefield Middlesex, UB9 6JH, UK (telephone: +44 (0)1895828977; fax: +44 (0)1895-828902; E-mail: secretariat@shvd.org; Website: www.shvd.org). 
The 2009 International Society

for Minimally Invasive

Cardiothoracic Surgery

(ISMICS) 12th Annual Scientific

Meeting

Meeting: June 3-6, 2009, Westin St. Francis in San Francisco, California. For more information, contact: EJ Weldon, 900 Cummings Center, Suite 221-U, Beverly, MA (Telephone: +1 978-927-8330; E-mail: ISMICS@prri.com).

Practices in Cardiac Surgery and Extracorporeal Technologies The New Orleans Conference

Conference: June 10-13, 2009, Westin Canal Place Hotel, New Orleans, Louisiana. For more information, contact: Joseph Basha, LP, CCP, CEO: Perfusion International, LLC, Program Director: The New Orleans Conference (Telephone: 1318-623-0890; E-mail: JosephBasha@ PerfusionInternational.com).

\section{European School for Cardio-}

Thoracic Surgery Cardiac

Course Level B

Meeting: June 15-20, 2009, Bergamo, Italy. For more information, contact: EACTS Executive Secretariat, 3 Park St., Windsor, Berkshire S14 1LU, UK (Telephone: +44-1753832-166; Fax: +44-1753-620407; E-mail: info@eacts.co.uk).

\section{The 5th World Congress of}

Paediatric Cardiology and

\section{Cardiac Surgery}

Congress: June 22-26, 2009, Cairns Convention Centre, Cairns, Queensland Australia. For more information, contact: ICMS Pty Ltd (Telephone: +61- 3- 9682- 0244; Email: pccs2009@icms.com.au).

The Western Thoracic Surgical

Association (WTSA) 35th

Annual Meeting

Meeting: June 24-29, 2009, Fairmont Banff Springs Hotel, Banff, AB Canada. For more information, contact:
April Conti; 900 Cummings Center; Suite 221-U, Beverly, MA 01915 (Telephone: +1 978-927-8330; FAX: +1 978-524-8890; Email:wtsa@prri.com).

\section{JULY 2009}

Heart \& Art: An Update on

Common Cardiovascular

Disorders

Conference: July 18, 2009, Danto Auditorium, University of Michigan Cardiovascular Center, Ann Arbor, Michigan. For more information, contact: Registrar, Office of Continuing Medical Education, University of Michigan Medical School, G1200 Towsley Center, 1500 E Medical Center Drive, SPC 5201, Ann Arbor, MI 48109-5201 (telephone: 734-7631400; fax: 734-936-1641).

\section{Rocky Mountain Valve \\ Symposium}

Symposium: July 23-24, 2009, The International Heart Institute of Montana, Saint Patrick Hospital, Missoula, Montana. For more information, contact: Timothy Descamps, $500 \mathrm{~W}$ Broadway, Suite 350, Missoula, Montana 59802 (telephone: 406-329-5668; fax: 406-329-5880; E-mail: TimD@ ihimontana.org). Additional information: http://www.rockymountainvalve symposium.org

\section{The 13th World Conference on Lung Cancer (WCLC 2009)}

Conference: July 31 - August 4, 2009, San Francisco, California, USA. Abstract Submission Deadline: March 13, 2009. For more information, contact: Khara Robertson, Conference Manager (telephone: 1604-681-2153, e-mail atwclc2009@ meet-ics.com; Web site: http://www. 2009worldlungcancer.org/).

\section{AUGUST 2009}

22nd Annual Cardiology Update Conference: August 21-23, 2009, Grand Hotel, Mackinac Island, Michigan. For more information, contact:
Registrar, Office of Continuing Medical Education, University of Michigan Medical School, G1200 Towsley Center, 1500 E Medical Center Drive, SPC 5201, Ann Arbor, MI 481095201 (telephone: 734-763-1400; fax: 734-936-1641).

\section{SEPTEMBER 2009}

European School for Cardio-

Thoracic Surgery Cardiac

Course Level C

Meeting: September 21-26, 2009, Bergamo, Italy. For more information, contact: EACTS Executive Secretariat, 3 Park St., Windsor, Berkshire S14 1LU, UK (Telephone: +44-1753-832166; Fax: +44-1753-620407; E-mail: info@eacts.co.uk).

\section{OCTOBER 2009}

Venice 2009 Arrhythmias - 11th International Workshop on

\section{Cardiac Arrhythmias}

Workshop: October 4-7, 2009, Fondazione Vini, Venice, Italy. Abstract submission deadline: April 7, 2009. For more information, contact: Rita Reggiani - Project Leader, Rimini, Italy (Telephone: +39- 0541- 305822305811; Fax: +39 -0541-305842; E-mail: infor@venicearrhythmias.org).

The $\mathbf{2 3}^{\text {rd }}$ European Association for Cardio-Thoracic Surgery (EACTS) Annual Meeting

Meeting: October 17-21, 2009, Austria Centre, Vienna, Austria. Abstract submission deadline: April 1, 2009. For more information, contact: EACTS Executive Secretariat, 3 Park St., Windsor, Berkshire S14 1LU, UK (Telephone: +44-1753-832-166; Fax: +44-1753-620407; E-mail: info@ eacts.co.uk).

Association of Thoracic and Cardiovascular Surgeons of Asia (ATCSA) 19th Biennial Congress 2009

Congress: October 25-28, 2009, Sheraton Grande Walkerhill Hotel, 
Seou, Republic of 1 Korea. Abstract submission deadline: June 1, 2009. For more information, contact: Ms. Hyo-Shin Nam, InSession International Convention Services, Inc (Phone: +82- 2 -3471 8555; Fax: +82-2-521-8683; E-mail: atcsa2009@ insession.co.kr)

\section{Vanderbilt Valve Symposium: 21st Century Techniques for Complex Valve Surgery}

Symposium: October 29-30, 2009, Nashville Marriott Hotel, Nashville, Tennesse. For more information, contact: Joan Lorber, Vanderbilt Heart, Department of Surgery, 1215 21st Avenue South, 5209 MCE, Nashville, TN 37232-8802 (Telephone:1- 615- 3437363; E-mail: joan.lorber@vanderbilt. edu).
NOVEMBER 2009

\section{European School for Cardio-} Thoracic Surgery Cardiac Course Level C

Meeting: November 9-14, 2009, Bergamo, Italy. For more information, contact: EACTS Executive Secretariat, 3 Park St., Windsor, Berkshire S14 1LU, UK (Telephone: +44-1753-832-166; Fax: +44-1753-620407; E-mail: info@ eacts.co.uk).

\section{The 5th International Meeting of} the Onassis Cardiac Surgery Center: Current Trends in Cardiac Surgery and Cardiology Meeting: November 12-14 2009, Athens Hilton Hotel, Athens, Greece. For more information, contact: Liana
Iliopoulou, Triaena Tours \& Congress, 206 Sygrou Avenue, 17672 Athens (Kallithea) Greece (Telephone: +30 -210- 7499353; Fax: +30- 2107705752; E-mail: lianae@triaenatours.gr).

\section{The International Joint Meeting} on Thoracic Surgery

Meeting: November 25-27, 2009, Auditorio Fomento de Trabajo Barcelona, Barcelona Spain. Abstract submission deadline: May 15, 2009. For more information, contact: Oriol Seto, ACTO SERVEIS, C/ Bonaire, 7; 08301 Mataro, Barcelona, Spain (Telephone: +34 -937- 552- 382; Fax: +34 -937- 552- 383; E-mail: thoracic.surgery@actoserveis.com). Additional information: http://www. thoracicsurgery2009.org 